\title{
Central Cementifying Fibroma of Mandible - A Case Report with Literature Review
}

\author{
Issrani $\mathrm{R}^{* 1}$, Mathur $\mathrm{S}^{1}$, Chandra $\mathrm{S}^{1}$ and Prabhu $\mathrm{N}^{2}$
}

${ }^{1}$ Department of Oral Medicine and Radiology, Saraswati Medical and Dental College, Lucknow, Uttar Pradesh, India ${ }^{2}$ Department of Oral and Maxillofacial Surgery, Saraswati Medical and Dental College, Lucknow, Uttar Pradesh, India

${ }^{*}$ Corresponding author: Issrani R, Department of Oral Medicine and Radiology, Saraswati Medical and Dental College, Lucknow, Uttar Pradesh, India, E-mail: dr.rakhi.issrani00@gmail.com

Citation: Issrani R, Mathur S, Chandra S, Prabhu N (2015) Central Cementifying Fibroma of Mandible - A Case Report with Literature Review. J Dent Oral Care Med 1(1): 104. doi: 10.15744/2454-3276.1.104

Received Date: February 16, 2015 Accepted Date: May 13, 2015 Published Date: May 15, 2015

\begin{abstract}
Cementifying fibroma is a rare benign fibro-osseous tumor belonging to the same category as fibrous dysplasia and cemento-ossifying dysplasia. Clinically it usually presents as a painless, slowly growing mass in the jaw and thus the lesion is frequently ignored by the patient until the growth produces a noticeable swelling and facial deformity. The current case is reported because of rarity of such lesions and the paucity of information concerning them in the dental literature. In this report we have described a 38-year-old male with central cementifying fibroma of the anterior mandible region with the swelling that had appeared one year back. Radiographic imaging such as intra-oral, occlusal and panoramic imaging had been performed. Histological analysis of the excised specimen was done. A final diagnosis of central cementifying fibroma was made after correlating the history, clinical and radiographic features, coupled with histopathology.
\end{abstract}

Keywords: Central cementifying fibroma; Fibro-osseous lesions; Mandible; Radiology

\section{Introduction}

In 1971, World Health Organization (WHO) categorized four types of cementum-containing lesions: fibrous dysplasia, ossifying fibroma, cementifying fibroma, and cemento-ossifying fibroma [1]. Cementifying fibroma is a rare fibro-osseous lesion that affects the jaw bones [2] and is considered to arise from undifferentiated cells of the periodontal ligament tissues that are capable of forming cementum, lamellar bone, and fibrous tissue [3]. It is most commonly seen between the third and fourth decades of life [4], and is more frequent in women than in men (5:1) [5-7]. They arise in the mandible in $62 \%$ to $89 \%$ of patients, $77 \%$ occurring in the premolar region [8]. Clinically, these tumors manifest as a slow-growing mass that is normally well delineated and asymptomatic though over time the lesion may become large enough to cause facial deformation [9]. Radiographically, this lesion shows a number of patterns depending on the degree of mineralization of the lesion and usually manifests as a well delineated unilocular lesion containing variable amounts of radiopaque material [10]. Histopathologically this lesion consists of a highly cellular, fibrous tissue that contains varying amounts of calcified tissue resembling cementum [11]. Treatment comprises surgical resection of the lesion with enucleation and curettage of the bone bed [9].

The current case is reported because of rarity of such lesions and the paucity of information concerning them in the dental literature. In this report we have described a 38-year-old male with central cementifying fibroma of the anterior mandible region with the swelling that had appeared one year ago. Radiographic imaging such as intra-oral, occlusal and panoramic imaging had been performed. Histological analysis of the excised specimen was done. A final diagnosis of central cementifying fibroma was made after correlating the history, clinical and radiographic features, coupled with histopathology. The postoperative follow up at one month revealed no signs of recurrence. This paper also offers a review of the literature on this lesion.

\section{Case Report}

A 38-year-old male patient reported to Department of Oral Medicine and Radiology, Saraswati Dental College, Lucknow, India with a chief complaint of slowly enlarging painless swelling in the left lower front region of the jaw since a year. It started as a small swelling which grew slowly causing noticeable facial deformity which rendered him to seek dental consult. The patient reported having suffered trauma in the same area two years ago. There was no history of trismus, pus discharge, fever or malaise. History of paresthesia, weight loss and loss of appetite were also negative. The medical, social and family histories were unremarkable. The review of systems and physical examination revealed no abnormal findings and all the vital signs were in the normal range.

\section{Clinical examination}

Extra-oral examination revealed a slight fullness at the left parasymphyseal region of mandible with a diffuse and smooth-surfaced uniform swelling measuring approximately $4 \times 3 \mathrm{cms}$ in size. The skin over the swelling was normal without any change in colour, sinus tract or discharge. On palpation, temperature over the swelling was not raised. The swelling was hard in consistency and non-tender. Left submandibular lymph nodes were palpable, single in number, mobile and non-tender. 
Intra-oral examination revealed a diffuse, smooth-surface and well- circumscribed swelling measuring approximately $4 \times 3 \mathrm{cms}$ in left mandibular anterior region extending antero-posteriorly from mesial surface of left mandibular central incisor to mesial surface of left mandibular first premolar. Superio-inferiorly it extended from the tooth \#31 to tooth \#34 to the buccal and lingual vestibule with complete obliteration of buccal vestibule. There was disto-lingual displacement of tooth \#33 (Figure 1). On palpation, the swelling was bony hard in consistency, non-tender, non-fluctuant, and non-compressible. Buccal and lingual cortical plates were expanded. The teeth in the affected area were not tender on percussion and no mobility could be demonstrated. Mucosa over the swelling appeared normal with no sinus tract or any discharge. Other dental findings were gingival recession with tooth \#33, carious tooth \#36 and generalized attrition with mild stains.

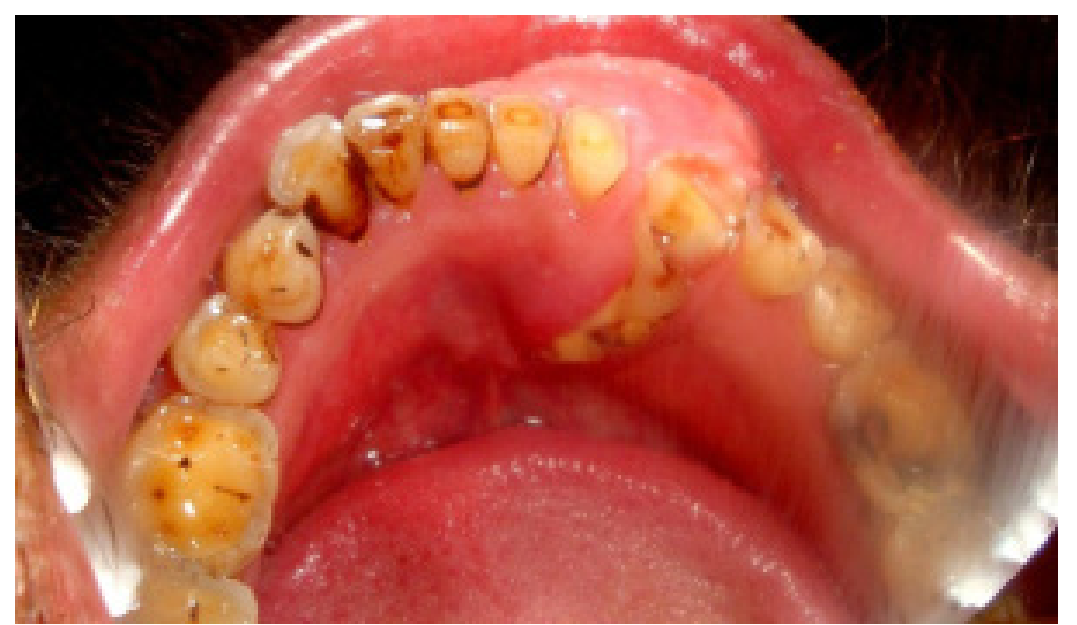

Figure 1: A large swelling on the left mandibular anterior region with disto-lingual displacement of tooth \#33

Depending upon the history and clinical examination a clinical provisional diagnosis of central giant cell granuloma was made. Differential diagnosis that was considered was cemento-ossifying fibroma, ameloblastoma and adenomatoid odontogenic tumour. The patient was subjected to following investigations to reach a probable diagnosis. Electric pulp vitality testing was done for tooth \#313233 34 which revealed all the teeth to be vital. A complete hemogram was also advised which showed all the values to be in the normal range.

Radiographic examination of intra-oral periapical radiograph in relation to tooth \#31 to tooth \#33 showed a hazy trabeculae and loss of lamina dura related to tooth \#32 and \#33 (Figure 2). Mandibular occlusal radiograph revealed a well-circumscribed large radiolucency with sclerotic border and foci of radiopacity in it measuring $4 \times 3 \mathrm{cms}$ buccally and $0.5 \times 0.5 \mathrm{cms}$ lingually involving mesial surface of tooth \#31 to mesial surface of tooth \#34 along with the reduced thickness of the buccal cortical plates with definite expansion (Figure 3). Orthopantomograph showed a hazy trabeculae and loss of lamina dura related to tooth \#32 and \#33 (Figure 4).

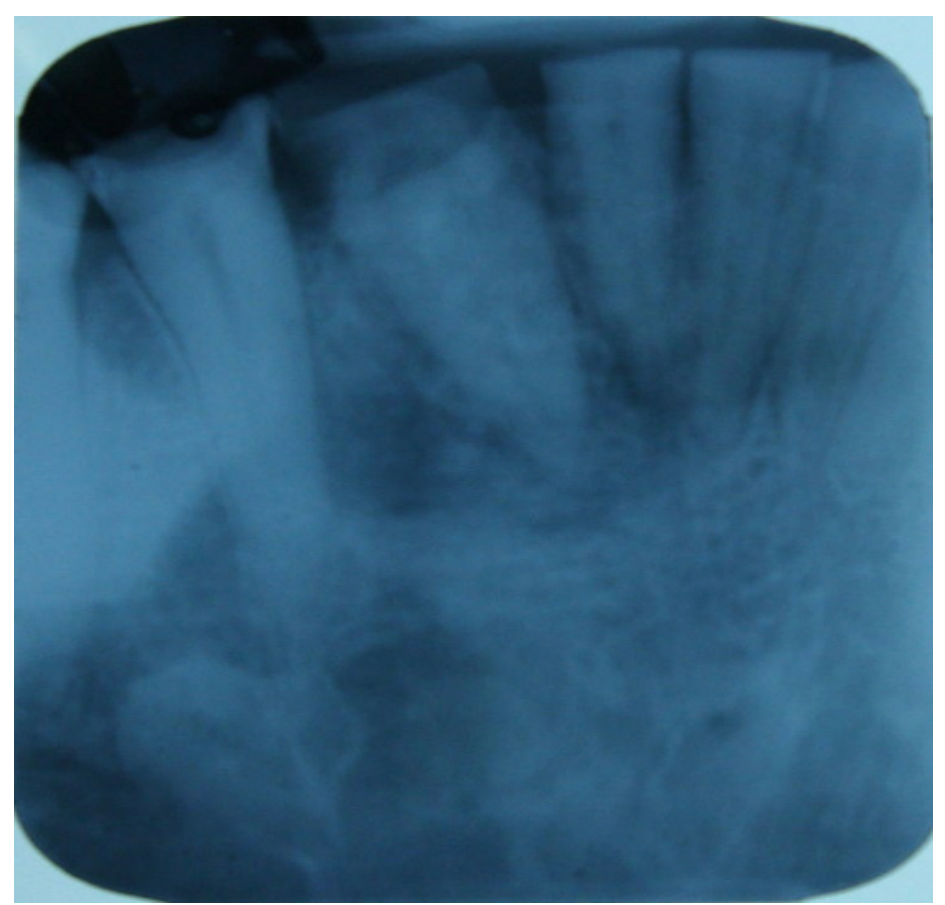

Figure 2: Intra-oral periapical radiograph in relation to tooth \#31 to tooth \#33 showing a hazy trabeculae and loss of lamina dura related to tooth \#32 and \#33 


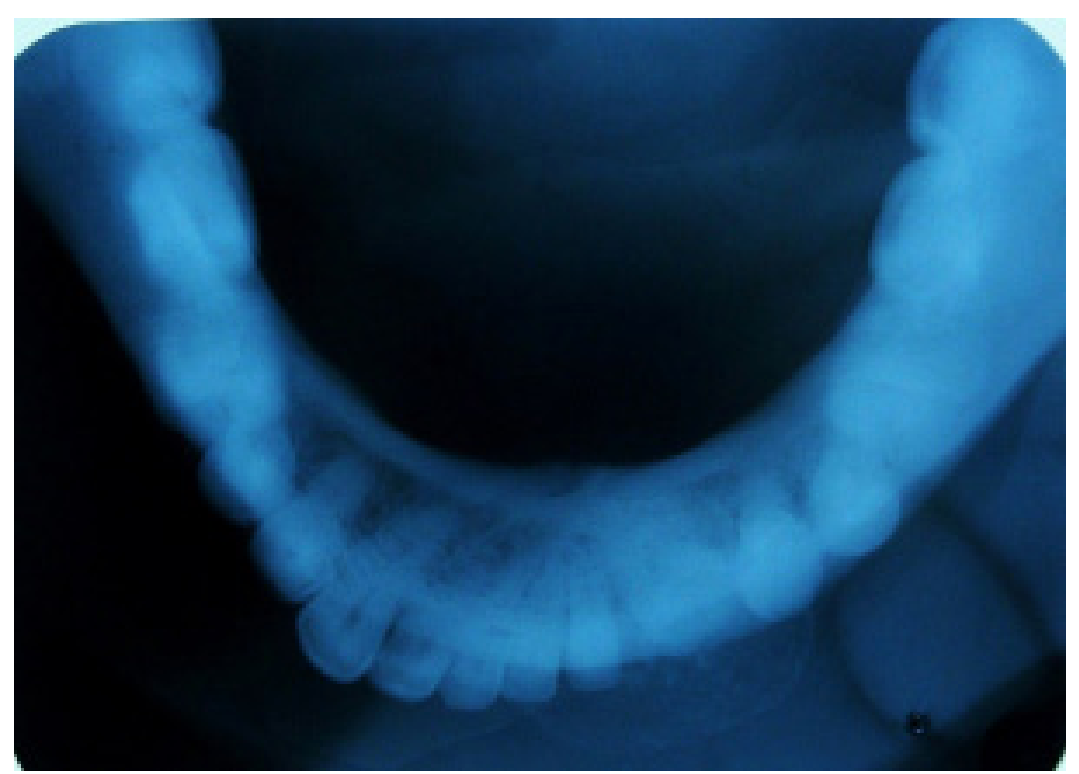

Figure 3: Mandibular occlusal radiograph revealing a well-circumscribed large radiolucency with sclerotic border and foci of radiopacity in it involving mesial surface of tooth \#31 to mesial surface of tooth \#34 along with the reduced thickness of the buccal cortical plates with definite expansion

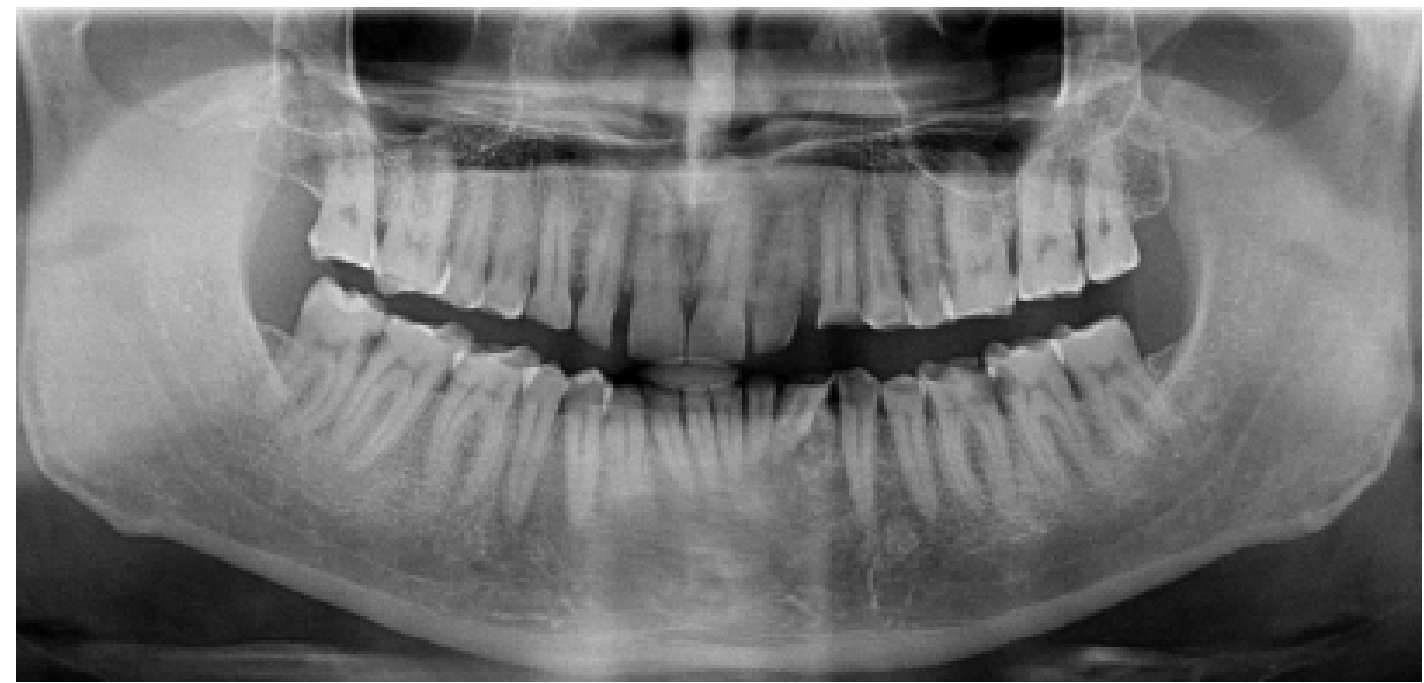

Figure 4: OPG showing a hazy trabeculae and loss of lamina dura related to tooth \#32 and 33

\section{Treatment}

Complete surgical removal of the lesion was carried out along with the tooth \#35 to \#42 and the adjacent bone. The gross specimen (Figure 5) was subjected to histopathological analysis.

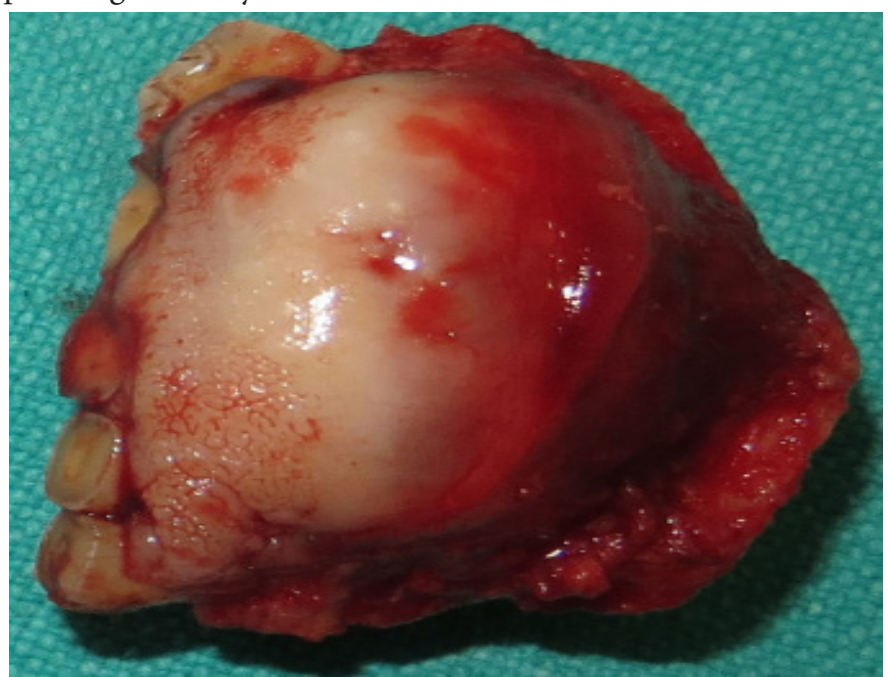

Figure 5: Completely excised lesion 


\section{Microscopic examination}

Microscopic examination of tissue section revealed a well encapsulated tumor mass of proliferating fibrous tissue with the cellular elements predominating. Several tiny spherical masses of darkly stained cellular calcified material resembling cementum was seen through the fibrous tissue and appeared in some region to be merging to form conglomerate masses. Presence of few blood capillaries and scanty cells were also evident. The above features were consistent with the diagnosis of central cementifying fibroma (Figure 6).

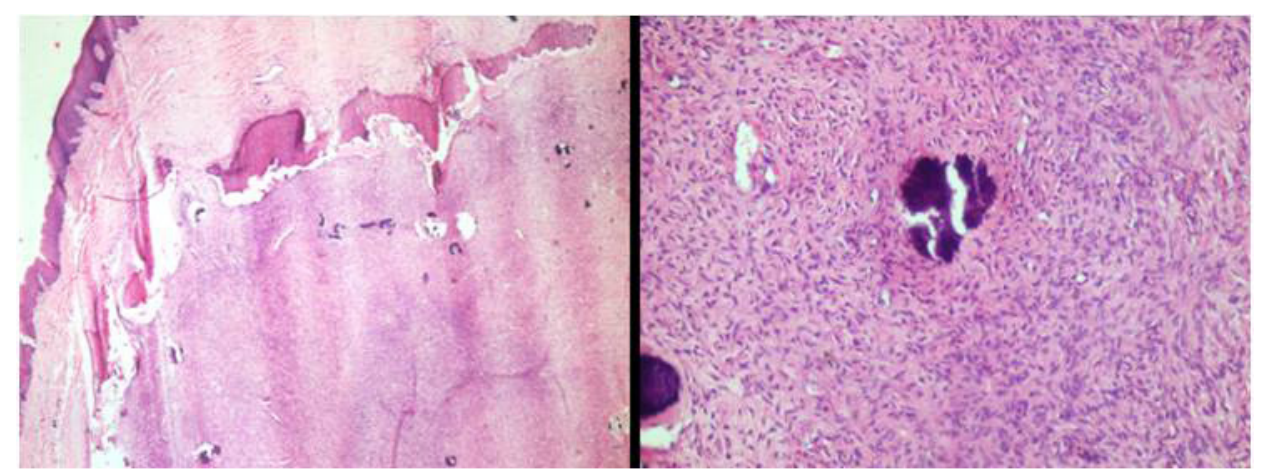

Figure 6: Histological analysis showing cemental tissue in the form of spherical masses in a fibrous stroma

\section{Postoperative process}

The patient was discharged from the hospital on the fourth postoperative day and was then seen at one week intervals. When the patient was last seen, there had been no recurrence of the lesion (Figure 7).

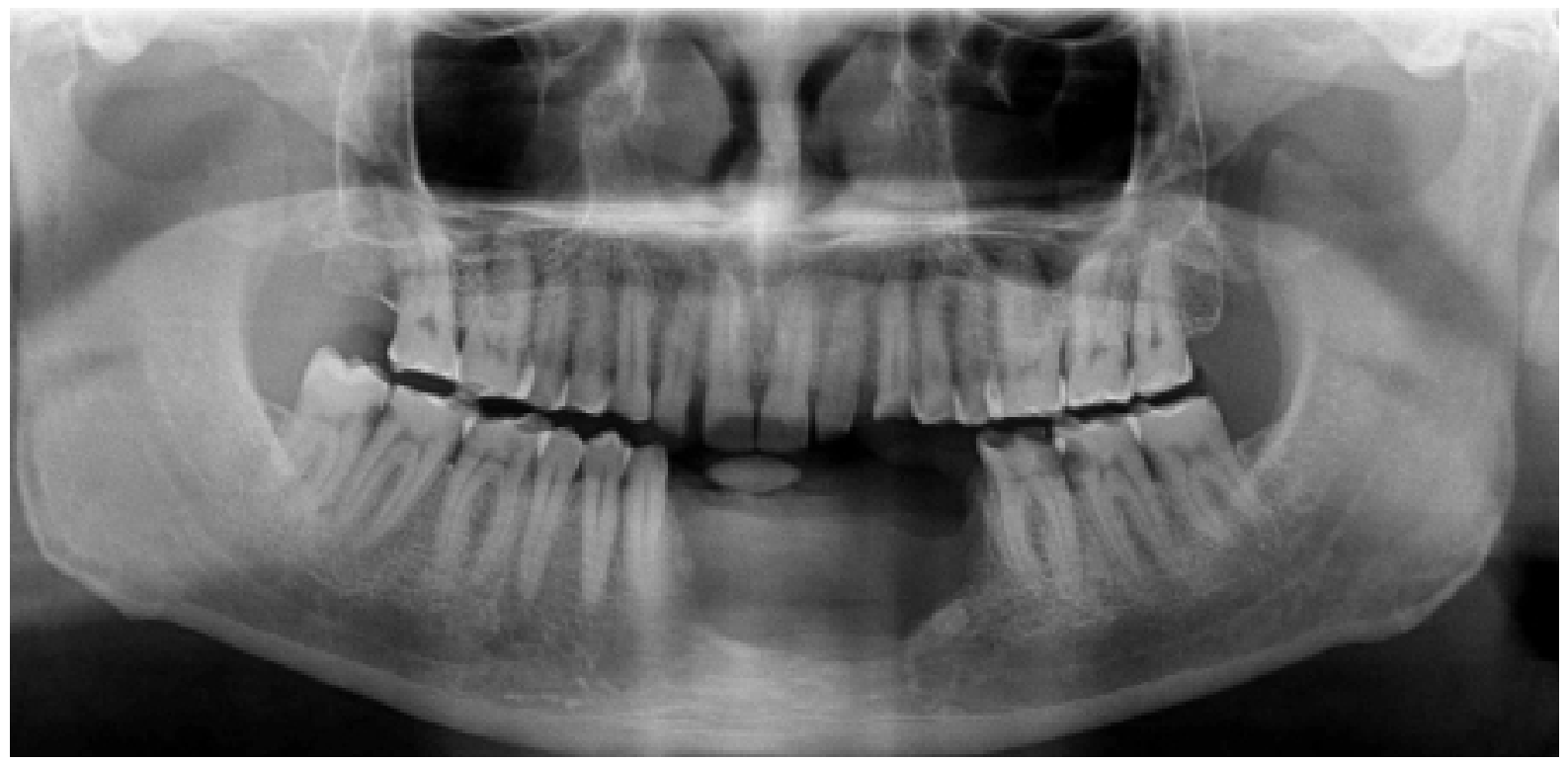

Figure 7: Post-operative OPG showing eventful healing of the excised area

\section{Discussion}

Fibro-osseous lesions comprise a group of disorders that are characterized by the replacement of normal bone by a benign connective-tissue matrix with varying amounts of mineralized substances [12]. They were initially classified into three main categories namely, fibrous dysplasia, fibro-osseous lesions such as ossifying and cementifying fibroma, and fibro-osseous neoplasms such as juvenile active ossifying fibroma. In recent years, these lesions were reclassified into fibrous dysplasia, reactive lesions arising in the tooth-bearing area, and fibro-osseous neoplasms such as cementifying and ossifying or cemento-ossifying fibroma [13]. Central cementifying fibroma is a distinct form of benign fibro-osseous lesions of the jaw bones [14]. A close histogenetic relationship exists between the central cementifying fibroma and the central ossifying fibroma. It is based on the marked similarity between the two regarding predilection of age of occurrence, sex, race, location, clinical behaviour, and roentgenographic appearance. The only difference between the two being in the type of cell involved and its end product-cementum in one case and bone in the other. This has prompted many to use the term cemento-ossifying fibroma [15].

Cementifying fibroma is thought to arise from the periodontal ligament and is composed of varying amounts of cementum, bone, and fibrous tissue [3]. Despite its origin in the periodontal membrane, the factors that stimulate this structure to produce cementum in an aberrant anatomical site remain controversial. Inflammation secondary to either infections or trauma has been 
proposed as a causative agent [16]. In coincidence with the data found in the literature, our patient reported having suffered trauma in the affected zone two years ago. This shows that trauma might be a possible triggering factor in some presentations of the lesion, postulating the cementifying fibroma as representing a connective tissue reaction rather than a neoplasm.

It commonly occurs between the third and fourth decade of life [4] and affects females more than males, with female-to-male ratios as high as 5:1 [5-7]. They are frequently found in the mandible than in the maxilla. In mandible, it occurs particularly in the premolar-molar region. $22 \%$ can be found involving molar region of maxilla, ethmoidal and orbital regions and is seen exceptionally in petrous bone [17]. When this arises in children, it has been named the Juvenile aggressive COF, which presents at an earlier age and is more aggressive clinically and more vascular at pathological examination [18]. Unlike these characteristic clinical features of cementifying fibroma, the present case is reported in a male patient involving the mandibular anterior region.

Clinically, the cementifying fibromas presents as a painless, and relatively slow growing mass in the jaw where displacement of teeth may be the only early clinical feature [19]. On rare occasions, however, pain or paresthesia may be elicited if pressure on an adjacent nerve ensues. Teeth in association with the lesion retain their vitality and, as a rule, there is no associated root resorption [18]. The lesion is therefore frequently ignored by the patient until the growth produces a noticeable swelling and facial deformity [19]. This seem to be the case in our patient considering that he has ignored the swelling in his jaw as it had been asymptomatic and that the lesion had all grown considerably big when he first came to our department. Cementifying fibroma often exhibit marked buccal and lingual bony expansion [18] and similar finding was present in our case.

The radiographic appearance is of utmost importance in the diagnosis of cementifying fibroma because it is often needed to separate it from other fibro-osseous lesions [20]. Radiographically, two basic patterns have been defined: one characterized by the presence of a unilocular or multilocular radiolucent image, and another showing mixed density due to a variable internal amount of radiopaque material. They are typically well circumscribed and maintain a spherical shape, expand the surrounding cortical bone without cortical perforation, and may cause tooth divergence [10]. In our case a mixed image was observed on the mandibular occlusal radiograph with expansion of buccal cortical bone. Interestingly, the differences in the amount of opacities within the lesion seem to have correlation with the duration of tumor themselves [10]. The tumor in our case was of one year duration and this long duration strongly suggest that the older the tumor the larger the amount of the calcified material found within the lesion. One additional important diagnostic feature radiographically is that there is a centrifugal growth pattern rather than a linear one and therefore the lesions grow by expansion equally in all directions and present as a round tumor mass [7]. This characteristic rounded-shape was reflected in our case reported herein. There are three different patterns of radiographic borders of cemento-ossifying fibroma which are: defined lesion without sclerotic border (40\%); defined lesion with sclerotic border (45\%); and lesion with ill-defined border (15\%) indicating a rapidly growing tumor [19]. In the present case, defined margins with sclerotic border were seen.

The characteristic macroscopic feature of this tumor is the presence of irregularly shaped calcifications within a hypercellular fibrous connective tissue stroma. The calcifications are extremely variable in appearance and represent various stages of cementum deposition. Histologic differentiation between osteoid and cementum is difficult. Most pathologists feel that central cementifying fibromas and central ossifying fibromas arise from the same progenitor cell but produce variable amounts of bone and cementum within any one lesion. The hybrid term central cemento-ossifying fibroma has evolved to indicate the likely presence of both types of tissue within the same lesion because of the difficulty in being able to distinguish reliably immature bone from immature cementum and because of the presence of both of these substances in many of the lesions. Thus, central cemento-ossifying fibroma is the most accurate histologic term, but it can be interchanged with either central ossifying fibroma or central cementifying fibroma. There is no apparent clinical or radiologic difference between the central cementifying fibroma and central ossifying fibroma, so the hybrid central cemento-ossifying fibroma works for radiology as well [3].

The differential diagnosis of cementifying fibroma includes chondrosarcoma or osteosarcoma, fibrous dysplasia, odontogenic cysts, squamous cell carcinomas, calcifying odontogenic cysts, and calcifying epithelial odontogenic tumors. The well-defined border of the central cementifying fibroma helps differentiate it from the aggressive sarcomas and carcinomas. Fibrous dysplasia has a characteristic "ground glass" appearance not seen in the central cementifying fibroma. The radiologic differentiation of central cementifying fibroma from calcifying odontogenic cysts and calcifying epithelial odontogenic tumors is difficult; the final diagnosis is based on histologic appearance [3].

The recommended treatment of the central cementifying fibroma is surgical excision. It usually "shell out" easily at surgery, but maxillary central cementifying fibroma is more difficult to remove completely than mandibular central cementifying fibroma. This may be attributable to the difference in bone character between the mandible and maxilla and to the available space for expansion in the maxillary sinus. Radiotherapy is contraindicated because of its radio resistance and post-radiation complications. The prognosis is usually good, since recurrences are not frequent. However, in some series, it has been reported to be as high as $28 \%$ [8].

This was confirmed in our case, since repair of the affected area was seen to be correct one month after treatment - though longer follow-up is required. 


\section{Conclusion}

The current case is reported because of rarity of such lesions and the paucity of information concerning them in the dental literature. We believe that this case illustrates many of the clinical, radiographic and histologic features associated with central cementifying tumours.

\section{References}

1. Liu Y, You M, Wang H, Yang Z, Miao J, et al. (2010) Ossifying fibromas of the jaw bone: 20 cases. Dentomaxillofac Radiol 39: 57-63.

2. Desai D, Arathi K, Ahmed S, Rai N (2012) Bilateral cemento ossifying fibroma of mandible. IJDA 2: 371-4.

3. Tamiolakis D, Thomaidis V, Tsamis I, Alexiadis G, Seretis K (2007) Clinical, radiological and histological correlation in the diagnostic work-up of cemento ossifying fibroma of the maxilla: apropos a case. Chirurgia (Bucur) 102: 359-62.

4. Mitra R, Baskaran P, Satyakumar M (2012) Imaging in the Diagnosis of Cemento ossifying Fibroma: A Case Series. J Clin Imaging Sci 2: 52.

5. Jung SL, Choi KH, Park YH (1999) Cemento ossifying fibroma presenting as a mass of the parapharyngeal and masticator space. AJNR Am J Neuroradiol 20: 1744-6.

6. Kuta AJ, Worley CM, Kaugars GE (1995) Central cemento ossifying fibroma of the maxillary sinus: A review of six cases. AJNR Am J Neuroradiol 16: 1282-6.

7. Sarwar HG, Jindal MK, Ahmad SS (2008) Cemento ossifying fibroma - A rare case. J Indian Soc Pedod Prev Dent 26: 128-31.

8. Hekmatnia A, Ghazavi A, Saboori M, Mahzouni P, Tayari N, et al. (2011). A case report of cemento ossifying fibroma presenting as a mass of the ethmoid sinus. J Res Med Sci 16: 224-8.

9. Silvestre-Rangil J, Silvestre FJ, Requeni-Bernal J (2011) Cemento ossifying fibroma of the mandible: Presentation of a case and review of the literature. J Clin Exp Dent 3: e66-9.

10. Bagi MA, Bokhari K, Nager MA, Basheer SA, Assiri MAM (2013) Cemento ossifying fibroma involving mandible. Int J Experimental Dent Sci 2: 127-9.

11. Gopinath D, Beena VT, Sugirtharaj G, Vidhyadharan K, Salmanul Faris K, et al. (2013) Cemento ossifying fibroma in a patient with end-stage renal disease. Case Rep Dent doi.org/10.1155/2013/923128.

12. Toyosawa S, Yuki M, Kishino M, Ogawa Y, Ueda T, et al. (2007) Ossifying fibroma vs fibrous dysplasia of the jaw: Molecular and immunological characterization. Modern Pathology 20: 389-96.

13. Ong AH, Siar CH (1998) Cemento ossifying fibroma with mandibular fracture. Case report in a young patient. Aust Dent J 43: $229-33$.

14. Godhi S, Goyal S, Giraddi G, Goyal S (2008) Cementifying Fibroma of the Mandible - A Case Report. J Oral Health Comm Dent 2: $42-5$.

15. Deshpande N, Munje SA, Wahane KD, Mantha S (2012) Cemento Ossifying Fibroma-A Rare Lesion in Pediatric Patients. Int J Dent Case Reports 2: 99-102.

16. Barberi A, Cappabianca S, Colella G (2003) Bilateral cemento ossifying fibroma of the maxillary sinus. Br J Radiol 76: 279-80.

17. Brademann G, Werner JA, Janig U, Mehdorn HM, Rudert H (1997) Cemento ossifying fibroma of petromastoid region: Case report and review of the literature. J Laryngol Otol 111: 152-5.

18. Bertrand B, Ely P, Cornelius JP, Gosseye S, Clotuche J, et al. (1993) Juvenile aggressive cemento ossifying fibroma: Case report and review of the literature. Laryngoscope 103: 1385-90.

19. Kamadjaja DB (2009) Cemento ossifying fibroma of the jaw. Dental journal 42: 164-71.

20. JayachandranS, Sachdeva S (2010) Cemento ossifying fibroma of mandible: Report of two cases. J Ind Acad Oral Med Radiol 22 : 53-6.

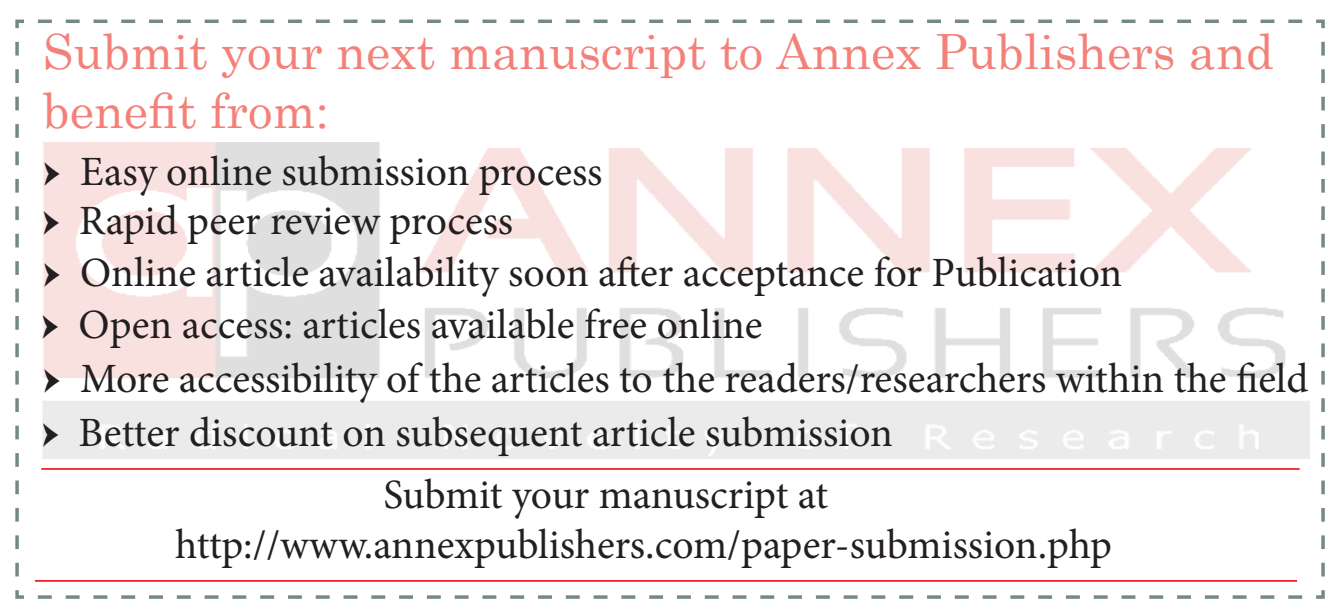

